\title{
Does Interleukin-2 Predict Success of Thrombolytic Therapy
}

\author{
Abdel Mohsen Mostafa Aboualia ${ }^{1 *}$, Eman Rashed Zaky ${ }^{1}$ and Mohamad Yousri Shaheen ${ }^{2}$ \\ ${ }^{1}$ Department of Cardiology, Al-Azhar University, Egypt \\ ${ }^{2}$ Department of Clinical pathology, Al-Azhar University, Egypt
}

*Corresponding author: Abdel Mohsen Mostafa Aboualia, Department of Cardiology, Al-Azhar University, Cairo, Egypt.

Received Date: June 05, 2020

Published Date: June 19, 2020

\begin{abstract}
Background: Atherosclerosis and its complication is proved to be an inflammatory process with increasing evidence of the role of cytokines in atherosclerosis pathophysiology.

Objective: To investigate the predictive role of admission level of interleukin-2 (IL-2) in acute myocardial infarction patients in detecting success of thrombolytic therapy.

Patients and Method: Forty patients with the diagnosis of ST-elevation myocardial infarction (STEMI) (20 patients diagnosed as STEMI with successful thrombolytic therapy and 20 patients diagnosed as STEMI with failed thrombolytic therapy) from those attending the Coronary Care Units in Cardiology department Al Hussein university hospital between April to May 2013 underwent echocardiography and baseline and after 3days follow up of IL-2 level.
\end{abstract}

Results: The age in STEMI success group was $44.7 \pm 9.41$ years while in the failed thrombolytic group $58.3 \pm 7.93$ years, hypertension (HTN) and diabetes mellitus (DM) were more prevalent in STEMI success group while smoking more in thrombolytic failed group. In the present study, Interleukin-2 was $274.3 \pm 104.2 \mathrm{pg} / \mathrm{ml}$ in STEMI success group and $215.1 \pm 49.4 \mathrm{pg} / \mathrm{ml}$ in STEMI failed group with no significant difference.

Conclusions: Admission level of serum IL-2 failed to predict the success of thrombolytic therapy

\section{Introduction}

Acute coronary syndrome (ACS) is the most common presentation of cardiovascular disease. Sensitive biomarkers improve the diagnosis of ACS among clinician leading to advancement in the management and reduction in mortality [1]. Till this time the superiority of primary percutaneous coronary intervention (PPCI) in those patient is evidence based. ST-elevation myocardial infarction (STEMI) patients presented in COVID-19 era or to hospitals without PCI facilities should receive thrombolytic therapy. Prediction of success is crucial to determine eligibility for thrombolytic therapy. Interleukin-2 (IL-2) has multiple functions, sometimes opposing to each other during an inflammatory response. IL-2 has osteoprotective effect on regulatory cells expansion. Also, IL-2 stimulates natural killer cells to proliferate and induces cytolytic activity when present at high levels and stimulates
B cells division and antibody production. So, it has a role in the atherosclerosis inflammatory process and its sequale [2]. Recent studies have shown that IL-2 has an action on atherosclerosis and remodeling after myocardial infarction in mice [3]. But there is lack of results about IL-2 as a predictor of thrombolysis success.

\section{Aim of the Work}

To investigate the predictive role of admission level of interleukin-2 (IL-2) in acute myocardial infarction patients in detecting success of thrombolytic therapy.

\section{Patients and Method}

This study was included (40) patients with the diagnosis of STEMI from those attending the Coronary Care Units in Cardiology 
department Al-Hussein university hospital, between April 2013 and August 2013.

\section{The studied population were classified into two equal groups based on thrombolysis success}

STEMI success group: ST segment elevation myocardial infarction group with successful thrombolytic therapy which included 20 patients.

STEMI failed group: ST segment elevation myocardial infarction group with failed thrombolytic therapy which included 20 patients.

Exclusion criteria: previous myocardial infarction or coronary intervention in the last three month, active inflammation, or active immunological diseases.

\section{Method}

After informed consent was taken, all patients underwent the following: -

1. Detailed history with special emphasis on: Age and gender, smoking, diabetes mellitus, hypertension, and dyslipidemia.

2. Clinical (general, cardiac) examination.

3. Laboratory work up including

a) Cardiac enzyme creatine kinase-myocardial band (CKMB) and troponin I to diagnose STEMI.

b) Serum interleukin-2: by ELISA at time of admission and after 7 days (Orgenium Laboratories' Tiilitie 3 FIN-01720 Vantaa FINLAND).

4. Resting 12 leads ECG to diagnose TSEMI based on fourth universal definition of myocardial infarction [4].

5. Resting conventional trans-thoracic echocardiography at time of admission with the patients in the left lateral decubitus. Images were obtained using Philips IE $33 \mathrm{X}$ machine. Recordings and calculations of Aortic root, Left atrium dimension, internal measurements of the LV and LV systolic function using biplane modified Simpson's methods [5].

6. The patients were classified according to the success of thromboolysis identified by - resolution of chest pain, accelerated idio-ventricular rhythm (AIVR), and ST segment Resolution (STR) $>50 \%$ in the lead with maximum ST elevation in pre-Thrombolytic ECG after 2 hours of streptokinase infusion [6].

\section{Statistical Analysis of the Data}

The data were analyzed statistically using IBM-SPSS-22 Table 3: Comparison between the groups regarded to risk factors.
(Statistical Package for Social Science version 22). Means and standard deviation were used to describe data distribution. t-test was used for comparison between groups. The test was considered significant if the probability ( $\mathrm{p}$-value) was less than 0.05 .

\section{Result}

The clinical findings of the patients

\section{Age}

The age was lower in STEMI success group than STEMI failed group ( $44.5 \pm 8.41$ versus $58.2 \pm 4.63$ years) and this difference is statistically significant $(\mathrm{p}$ value $=0.01)$ (Table 1$)$.

Table 1: Comparison between the groups regarded to age (years).

\begin{tabular}{|c|c|c|c|}
\hline Age & STEMI Success & STEMI Failed & P Value \\
\hline Mean \pm SD & $44.5 \pm 8.41$ & $58.2 \pm 4.63$ & 0.01 \\
\hline
\end{tabular}

\section{Gender}

No statistically significant difference between the groups as regard gender (21 patients $(70 \%)$ were males in STEMI success group and 24(80\%) in STEMI failed group) with P value $>0.05$.

\section{Risk factors}

Regarding to the risk factors, in STEMI success group 10(50\%) of the patients had diabetes mellitus, 16(90\%) had hypertension and $10(50 \%)$ were smoker and in STEMI failed group $7(35 \%)$ of the patients had diabetes mellitus, $8(40 \%)$ had hypertension and $10(50 \%)$ were smoker. There was a statistically significant differences between the two groups as regard diabetes mellitus and hypertension (Table 2).

Table 2: Comparison between the groups regarded to risk factors.

\begin{tabular}{|c|c|c|c|c|c|}
\hline \multirow{2}{*}{$\begin{array}{c}\text { Risk } \\
\text { factors }\end{array}$} & \multicolumn{2}{|c|}{ STEMI Success } & \multicolumn{2}{c|}{ STEMI Failed } & \multirow{2}{*}{ P Value } \\
\cline { 2 - 5 } & No. & $\%$ & No. & $\%$ & \\
\hline DM & 15 & 50 & 10 & 33.3 & $0.01^{*}$ \\
\hline HTN & 27 & 90 & 12 & 40 & $0.01^{*}$ \\
\hline Smoker & 15 & 50 & 15 & 50 & $>0.05$ \\
\hline
\end{tabular}

Interleukin-2 level at time of admission and after 3 days: (pg/dl)

Regarding to the Interleukin-2, in STEMI success group it ranged between 140-475 pg/dl and mean \pm SD was $274.3 \pm 104.2$ $\mathrm{pg} / \mathrm{dl}$ and in STEMI failed group it ranged between $146-290 \mathrm{pg} / \mathrm{dl}$ and mean \pm SD was $215.1 \pm 49.4 \mathrm{pg} / \mathrm{dl}$. After 3 days, in STEMI success group mean \pm SD was $270.6 \pm 104.1$ and in STEMI failed group mean \pm SD was $218.9 \pm 49.8$. There was no statistically significant difference between the four groups with $\mathrm{P}>0.05$ (Figure 1 \& Table $3)$.

\begin{tabular}{|c|c|c|c|c|c|}
\hline \multirow{2}{*}{ Interleukin-2 } & \multicolumn{2}{|c|}{ STEMI Success } & \multicolumn{2}{c|}{ STEMI Failed } & \multirow{2}{*}{ P value } \\
\cline { 2 - 6 } & At time of admission & After 7 days & At time of admission & After 7 days & \\
\hline Mean \pm SD & $274.3 \pm 104.2$ & $270.6 \pm 104.1$ & $215.1 \pm 49.4$ & $218.9 \pm 49.8$ & $>0.05$ \\
\hline
\end{tabular}




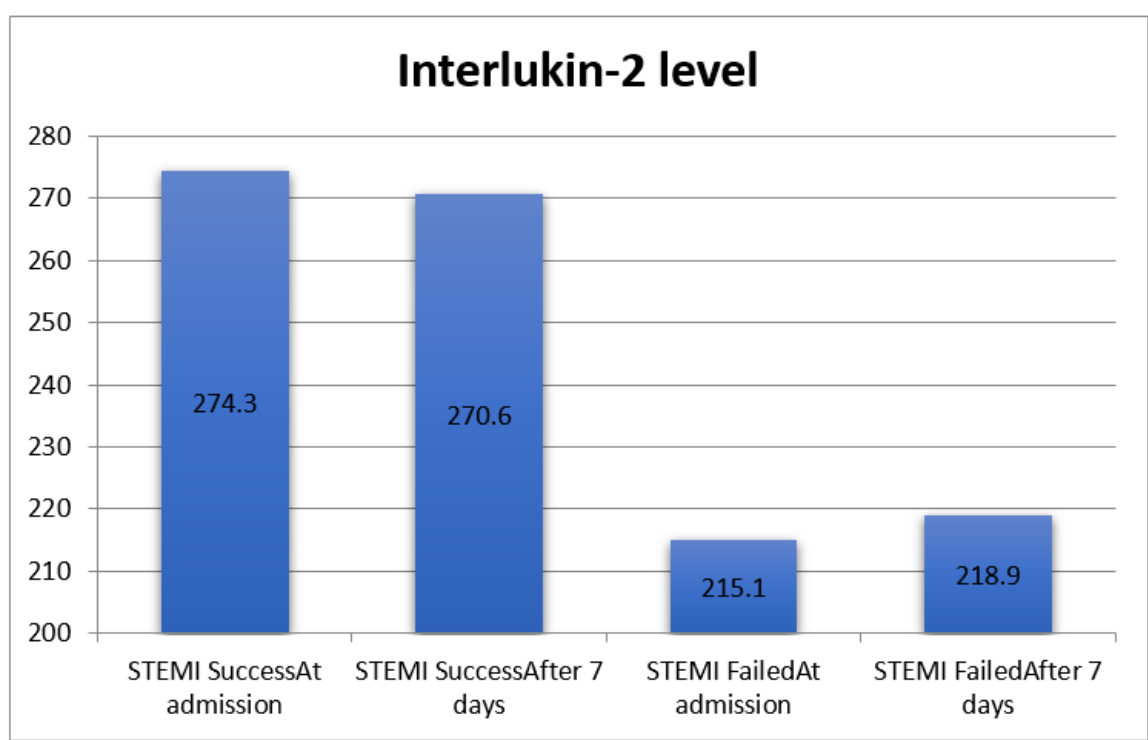

Figure 1: Comparison between the groups regarded tolnterleukin-2.

\section{Echocardiographic parameters}

No significant difference between the two groups regarding conventional echo-parameters. Global longitudinal strain of LV was reduced in both groups and more in STEMI failed group although this difference is not significant ( $p$ value $>0.5$ ) (Table 4).

Table 4: Echo parameters of the two groups at time of admission.

\begin{tabular}{|c|c|c|c|}
\hline Variable & STEMI Success & STEMI Failed & P Value \\
\hline Aortic diameter & $32.51 \pm 4.22$ & $31.21 \pm 4.53$ & $>0.05$ \\
\hline Left atrium diameter & $38.20 \pm 2.0$ & $35.6 \pm 3.70$ & $>0.05$ \\
\hline Interventricular septum diameter at diastole & $8.0 \pm 0.1$ & $8 \pm 0.2$ & $>0.05$ \\
\hline Left ventricular internal dimension at diastole & $53.3 \pm 2.2$ & $52.3 \pm 2.5$ & $>0.05$ \\
\hline Posterior wall thickness at diastole & $7.0 \pm 1.2$ & $7.0 \pm 1.5$ & $>0.05$ \\
\hline Interventricular septum diameter at systole & $12.0 \pm 2.2$ & $12.1 \pm 3.1$ & $>0.05$ \\
\hline Left ventricular internal dimension at systole & $35.6 \pm 2.4$ & $36.3 \pm 1.2$ & $>0.05$ \\
\hline Posterior wall thickness at systole & $11.3 \pm 1.1$ & $13.5 \pm 1.7$ & $>0.05$ \\
\hline Ejection fraction & $50.2 \pm 3.2$ & $49.8 \pm 5.2$ & $>0.05$ \\
\hline Global longitudinal strain & $-18.2 \pm 2.4$ & $-17.9 \pm 3.1$ & $>0.05$ \\
\hline
\end{tabular}

\section{Discussion}

There has been more evidence of the role of immune and inflammatory processes in the development and progression of atherosclerosis. Macrophages and T lymphocytes have many roles one of them release of cytokines [7]. Also, nowadays increasing roles of targets therapy for atherosclerosis made plenty of cytokines research [8]. Interleukin-2 (IL-2) is a small $\alpha$-helical cytokine that regulates immune cell homeostasis and discovered more than 30 years earlier. IL-2 showed to have therapeutic efficacy for immune diseases by its anti-inflammatory action mediated by regulatory $\mathrm{T}$ cell expansion. Combining this finding with the pathophysiology of myocardial infarction in the form of plaque rupture and erosions elucidate the protective role of this cytokine in myocardial infarction [9].
In Dinh, et al. [10] the IL-2 complex ( composed of IL-2 and anti-IL-2 monoclonal antibody ) was found to selectively expands the athero-protective regulatory T cells up to 6 times and effectively inhibits the development and progression of atherosclerosis in Apolipoprotein E -/- mice. So, significantly attenuates the ventricular remodeling and resulting in reduced infarct size with improved left ventricular (LV) function after myocardial infarction. Also, Webster, et al. [11] demonstrated that IL-2 attenuated cardiomyocyte apoptosis in a mouse myocardial infarction model. Zeng, et al. [3] demonstrated that IL-2 complex exerts cardiac protective effects by directly inhibiting the infiltration of macrophages and facilitating the polarization of anti-inflammatory M2 macrophages, which results in attenuation of cardiomyocytes apoptosis and the inflammatory responses. The expansion of regulatory $\mathrm{T}$ cells by the 
IL-2 complex may be a potentially valuable approach in improving ischemic heart disease.

In Bouchentouf, et al. [12] found that a single intravascular injection of recombinant human IL-2(rhIL-2) two days after myocardial infarction improved the ejection fraction of left ventricular by $27.7 \%$ of immune competent mice but no effect on the ejection fraction of the immune-deficient mice. Cardiac angiogenesis and decreased collagen deposition were enhanced by the administration of rhIL-2 and this help to preserve the integrity and function of myocardium especially in the scar and border areas of infracted myocardium. In this study no gender difference between the groups with diabetes mellitus and hypertension were more prevalent in STEMI success group. In Ding, et al. [7] hypertension more prevalent in CAD group (46.5\%).

In the current study, patients were presented by STEMI and underwent thrombolysis; half of them respond successfully to thrombolytic therapy. Although the level of interlukin-2 was lower in failed to respond to thrombolytic group at baseline and after 3 days this difference was non-significant statistically. In this study no role for echocardiography in prediction of outcome of thrombolytic therapy and no previous known study addressed this issue. The elevation of these markers in STEMI success group may be explained by the release of metabolites after pharmacological revascularization. IL-2 may be induced in the repercussed myocardium to modulate the reaction to injury. In other words, increased levels of IL-2 suggest good reperfusion, while low of IL-2 level means ischemic but not repercussed myocardium.

Ding, et al. [7] found an increased IL-2 expression in CAD patients $(39 \mathrm{pg} / \mathrm{ml})$ than in healthy controls $(27 \mathrm{pg} / \mathrm{ml})(\mathrm{p}<0.01)$ but they did not risk stratify the patients. This study is the first to study interlukin2 as a predictor for successful thrombolysis in acute myocardial infarction patients.

\section{Conclusion}

Admission level of serum IL-2 failed to predict the success of thrombolytic therapy and we must search for another cytokine as a predictor.

\section{Acknowledgement}

None.

\section{Conflict of Interest}

No conflict of interest.

\section{References}

1. Giugliano RP, Braunwald E (2011) The year in non-ST-segment elevation acute coronary syndrome. J Am Coll Cardiol 58(22): 2342-2354.

2. Ye C, Brand D, Zheng SG (2018) Targeting IL-2: an unexpected effect in treating immunological diseases. Signal Transduct Target Ther 3: 2 .

3. Zeng Z, Yu K, Chen L, Li W, Xiao H, et al. (2016) Interleukin-2/antiinterleukin-2 immune complex attenuates cardiac remodeling after myocardial infarction through expansion of regulatory T cells. J Immunol Res 2016: 8493767.

4. Thygesen K, Alpert JS, Jaffe AS, Simoons ML, Chaitman BR, et al. (2012) Third Universal Definition of Myocardial Infarction. Circulation 33(20): 2551-2567.

5. Lang RM, Badano LP, Mor-Avi V, AfilaloJ, Armstrong A, et al. (2015) Recommendations for cardiac chamber quantification by echocardiography in adults: an update from the American Society of Echocardiography and the European Association of Cardiovascular Imaging. J Am Soc Echocardiogr 28(1): 1-39.

6. Kuchulakanti PK (2009) Rescue PTCA--current status. Indian Heart J 61(5): 462-463

7. Ding R, Gao W, Ostrodci DH, He Z, Song Y, et al. (2013) Effect of interleukin-2 level and genetic variants on coronary artery disease. Inflammation 36(6): 1225-1231.

8. Zhao TX, MallatZ (2019) Targeting the Immune System in Atherosclerosis. JACC 73(13): 1691-1706.

9. Ye C, Brand D, Zheng SG (2018) Targeting IL-2: an unexpected effect in treating immunological diseases. Signal Transduct Target Ther 8(3): 2

10. Dinh TN, Kyaw TS, Kanellakis P, Kelly To, Peter Tipping, et al. (2012) Cytokine therapy with interleukin-2/anti-interleukin-2 monoclona antibody complexes expand CD4+CD25+Foxp3+ regulatory $\mathrm{T}$ cells and attenuates development and progression of atherosclerosis. Circulation 126: $1256-1266$.

11. Webster KE, Walters S, Kohler RE, Tomas Mrkvan, Onur Boyman, et al. (2009) In vivo expansion of T reg cells with IL-2-mAb complexes: induction of resistance to EAE and long-term acceptance of islet allografts without immunosuppression. J Exp Med 206: 751-760.

12. Bouchentouf M, Williams P, Forner KA, J Cuerquis, V Michaud, et al. (2011) Interleukin-2 enhances angiogenesis and preserves cardiac function following myocardial infarction. Cytokine 56: 732-738. 